\title{
Genes in the Azoospermia Factor A Region of the $Y$ Chromosome Show Sexual Dimorphism in Rat Brain Prior to Gonadal Sex Differentiation
}

\section{Ajay Pradhan}

Örebro Universitet: Orebro Universitet

\section{Subrata Pramanik}

Örebro Universitet: Orebro Universitet

Per-Erik Olsson ( $\square$ per-erik.olsson@oru.se)

Örebro universitet Akademin för Naturvetenskap och Teknik https://orcid.org/0000-0001-7336-6335

\section{Research Article}

Keywords: Brain sex, sex chromosome, azoospermia factor A, neurons, RNA sequencing, sexual dimorphism

Posted Date: December 28th, 2021

DOI: https://doi.org/10.21203/rs.3.rs-1179896/v1

License: (c) (i) This work is licensed under a Creative Commons Attribution 4.0 International License. Read Full License 


\section{Abstract}

\section{Background}

The classical concept of brain sex differentiation suggests that steroid hormones released from the gonads program male and female brains differently. However, several studies indicate that steroid hormones are not the only determinant of brain sex differentiation and that genetic differences could also be involved.

\section{Methods}

In this study, we have performed RNA sequencing of rat brains at embryonic days 12 (E12), E13, and E14. The aim was to identify differentially expressed genes between male and female rat brains during early development.

\section{Results}

Analysis of genes expressed with the highest sex differences showed that Xist was highly expressed in females having XX genotype with an increasing ratio over time. Analysis of genes expressed with the highest male expression identified three main genes. At E12, two genes located in the azoospermia factor A (AZFa) region on the $Y$ chromosome were highly expressed in males. These were $D d x 3 y$ (1552-fold higher in males) and $K d m 6 c$ (147-fold higher in males). The expression of $K d m 6 c$, but not $D d x 3 y$, remained high at both E13 and E14. In qRT-PCR analysis, these two genes were highly expressed in all the stages in male brain. In addition to these genes, one of the several copies of Sry in the rat genome, Sry4, showed a high expression in the male brains at all three time points. At all three time points several other genes were also found to show sex bias, but with lower differences in gene expression.

\section{Conclusion}

The observed sex-specific expression of genes at early development suggests that the rat brain is sexually dimorphic prior to gonadal action on the brain and identifies the AZFa region genes as a possible contributor to male brain development.

\section{Introduction}

Sexual dimorphism, including maternal care, sexual behavior, brain function, structure and susceptibility to neurological disorders are evident in humans as well as in nonhuman species. Earlier studies suggest that human male and female brains display differential connectome, methylome and transcriptome profiles (Ingalhalikar et al. 2014, Xu et al. 2014). Despite extensive advancement in neuroscience, the molecular regulation of these sex differences remains unclear. The classical model of brain sex differentiation that placed gonadal steroid hormones as the main drivers in establishing male and female neural networks was derived from earlier studies (Phoenix et al. 1959, Arnold 2009). This model states that the chromosomal constitution ( $\mathrm{XX}$ or $\mathrm{XY}$ ) determines the gonadal sex and that hormones secreted by 
these programs the brain neural network differently (Phoenix et al. 1959, Arnold 2009). However, a study on mice indicated that the developing whole head displayed differential gene expression prior to gonadal hormone action on the brain (Dewing et al. 2003). In that study, DEAD-box RNA helicase $y$ ( $d d x 3 y$, dby) and eukaryotic translation initiation factor 2 subunit 3 y (Eif2s3y) were identified as having the highest male biased gene expression at $10.5 \mathrm{dpc}$ (days post coitum), while $X$-inactive specific transcript (Xist) showed the highest female bias. Sex determining region $Y(S r y)$ is the master regulator gene located on $Y$ chromosome that determines testis fate (Koopman 2005). The expression of Sry can be detected at around $10.5 \mathrm{dpc}$ in mouse gonads and peaks at $11.5 \mathrm{dpc}$ to initiate testis differentiation (Sim et al. 2008). A similar study in chicken also showed that male and female brains are sexually dimorphic prior to gonadal development (Lee et al. 2009). This indicates that there are genetic sex differences in developing brains prior to hormone action and suggests that these differences may be involved in the differential development of male and female brains.

Aromatase inhibitor treatment, that induced testicular tissue in the genetic female zebra finch, failed to masculinize the song system that remained feminine (Wade and Arnold 1996). Another study on zebra finch showed that androgen treatment of female birds did not fully masculinize the song center (Gahr and Metzdorf 1999). Furthermore, an involvement of genetic factors in brain sex development was suggested in a study of a gynandromorphic zebra finch that had one half of its brain and body genetically male and the other half genetically female (Arnold 2003). Despite the whole brain being under the influence of the same gonadal hormones, the zebra finch still developed histologically identifiable song centers in the male side of the brain while the female side of the brain remains feminine (Arnold 2003).

In the rat, it has been determined that Sry expression peaks on day 13, suggesting that this is the time when testis differentiation is initiated (Prokop et al. 2020). Therefore, we selected rat prenatal brains at E12, E13 and E14 to perform RNA sequencing to identify differentially expressed genes prior to the initiation of hormonal effects on the brain. At E12, prior to the initiation of testis development, we identified 2 genes located to the azoospermia factor $\mathrm{A}$ (AZFa) region on the $\mathrm{Y}$ chromosome, $D d x 3 y$ and $\mathrm{N}^{\epsilon}$-methyl lysyl demethylase $(K d m 6 c)$, that may be involved in male brain sex differentiation. As Sry 4 showed sex biased gene expression at all three developmental stages, locally expressed SRY cannot be excluded as a master regulator of gene expression in the brain prior to testis differentiation.

\section{Materials And Methods}

\section{Sample processing, RNA sequencing and analysis}

Brain samples from Rattus norvegicus (Sprague Dawley) were purchased from Brain Bits USA. The samples were homogenized in Tri Reagent (Sigma) and RNA extraction was performed using Directzol RNA extraction kit (Zymo Research, USA). RNA was quantified using nano drop (Denovix, USA) and the quality was analyzed using RNA denaturing gel. RNA samples were sent to GATC Biotech/Eurofins for RNA sequencing. Sequencing was performed using Illumina platform with 30 million reads. RNA 
sequencing data was analyzed using Partek Flow software (Partek, USA). The raw data files were first analyzed for sequence quality using pre-alignment QA/QC. The reads were aligned to rat genome (Rnor_6.0) using BWA-MEM alignment algorithm. The counts were normalized using counts per million reads method (CPM) and the differentially regulated genes were identified using gene specific analysis (GSA). A $p$ value of $p<0.05$ was used to identify significantly differentially expressed genes (DEGs). The regulated genes were then used for gene set enrichment analysis (GSEA) based on Gene Ontology (GO) annotations. GO enrichment analyses were performed with a $p$ value threshold of 0.05 .

\section{Genotyping}

For genotyping, tissue sample from the body was taken and genomic DNA was extracted using DNA isolation kit (Zymo Research, USA). DNA was quantified using nano drop (Denovix, USA) and PCR was performed for the Sry 4 gene. Beta actin was used as positive control. Primer sequences are listed in Table S1. The PCR reaction conditions were as follows: $95^{\circ} \mathrm{C}$ for 5 mins followed by 35 cycles of $95^{\circ} \mathrm{C}$ for 10 secs, $55^{\circ} \mathrm{C}$ for $15 \mathrm{sec}$ and $72^{\circ} \mathrm{C}$ for $1 \mathrm{~min}$. The PCR product was run on $1 \%$ agarose gel.

\section{Quantitative real-time PCR (qRT-PCR) validation}

Equal amounts of total RNA, from male and female brains at E12 to E14, were converted to CDNA using qScript cDNA synthesis kit (Quanta Biosciences, USA) according to the manufacturer's instructions. qRTPCR was performed on a CFX96 Real-Time PCR Detection System using SsoAdvanced SYBR Green (BioRad, USA). Thermocycling conditions for SYBR Green qRT-PCR consisted of an initial denaturation at $95^{\circ} \mathrm{C}$ for 2 mins, followed by 40 cycles of $95^{\circ} \mathrm{C}$ for 2 secs and $60^{\circ} \mathrm{C}$ for 30 secs. Gapdh was used as the normalizing control. For primer specificity, melting curves were analyzed and the PCR product was run on agarose gel. Quantitative data analysis of relative gene expression was performed using the $\Delta \Delta \mathrm{Ct}$ method (Schmittgen 2008). Primer sequences are listed in Table S1.

\section{Statistical analysis}

For the transcriptomics data, differentially expressed genes were considered statistically significant if $p$ values were $\leq 0.05$. Pathway enrichment analysis and gene set enrichment analysis were performed using differentially genes and were considered statistically significant if $p$ values were $\leq 0.05$.

Student's t-test was performed for qRT-PCR data using the GraphPad Prism 8 software (GraphPad software). The differences were considered significant when the $p$ value was $<0.05(* p<0.05 ; * * p<$ $0.01)$.

\section{Results}

\section{Gene expression patterns during rat brain development}


The three developmental stages, from E12 to E14, were compared to identify genes that could be involved in sex differentiation of the brain. Principal component analysis (PCA) showed that the overall gene expression in male and female brains from the three different stages clustered according to embryonic stages rather than sex (Figure 1A). The PC1 explained $43.19 \%$ of the variability, while PC2 and PC3 explained $12.67 \%$ and $8.2 \%$ of the variability, respectively.

Within each developmental stage, sexual dimorphic gene expression patterns were identified. In E12 brains, the expression of 13,440 genes was identified in male and female brains. Of these, 91 genes were upregulated in males, whereas 91 genes were upregulated in females (Figure 1B). In the E13 brains, 13,979 genes were expressed in male and female brains (Figure 1C). Of these, 80 genes were upregulated in male brain, whereas 349 genes were upregulated in female brains. In the E14 brains, the expression of 14043 genes was identified in male and female brains, of which 144 genes were upregulated in males and 368 genes were upregulated in females (Figure 1D). The increased number of differentially expressed genes indicates that the sex differences in the brain increased with developmental time.

\section{Differentially expressed genes in rat brains}

Analysis of the sex-specific gene expression patterns revealed that a substantial number of genes were upregulated in male brain already at E12 (Figure 2A, Supplementary Table 1). The highest male sex biased gene expression (difference between sexes exceeding 3-fold) at E12 was observed for $D d x 3 y$, Kdm6c, Sry4 and Calcitonin related polypeptide alpha (Calca), while the highest female sex biased gene expression (difference between sexes exceeding 3-fold) was observed for Xist, Somatostatin (Sst), Fibroblast growth factor 18 (Fgf18) and Cytochrome P450 family 26 subfamily B member 1 (Cyp26b1). At E13, Kdm6c and Sry 4 expression continued to show male sex bias while Xist, AABR07021574.1, Cellular retinoic acid binding protein 1 (Crabp1), Paired box 7 (Pax 7 ) and Corin showed female sex bias (Figure 2B, Supplementary Table 2). Finally, at E14, both $K d m 6 c$ and Sry 4 continued to exhibit high male biased gene expression while Xist remained female biased (Figure 2C, Supplementary Table 3). The highest fold difference was observed for Ddx3y at E12 (1,552-fold) and Kdm6c at E13 (134-fold) and E14 (200 fold) (Table 1). 
Table 1

Sexually dimorphic key gene expression in E12, E13, and E14 rat brains. Positive fold changes of gene expression indicate upregulation in the male rat brains, whereas negative fold changes of gene expression indicate upregulation in the female rat brains.

\section{(A) Identified sexually dimorphic key gene expressed in E12 rat brains}

\begin{tabular}{|llll|}
\hline Gene & Fold & Chr & Position (strand) \\
\hline Ddx3y & $1,552.15$ & Y & $1,217,800-1,238,454(-)$ \\
\hline Kdm6c & 147.41 & Y & $1,065,274-1,206,127(-)$ \\
\hline SRY4 & 5.02 & Y & $327,176-327,685(+)$ \\
\hline Calca & 3.48 & 1 & $184,184,018-184,188,922(-)$ \\
\hline Cyp26b1 & -3.09 & 4 & $116,261,796-116,278,615(-)$ \\
\hline Fgf18 & -3.17 & 10 & $18,047,109-18,082,290(-)$ \\
\hline Sst & -6.33 & 11 & $80,358,172-80,359,449(+)$ \\
\hline Xist & -14.85 & $X$ & $74,324,637-74,342,152(-)$ \\
\hline
\end{tabular}

(B) Identified sexually dimorphic key gene expressed in E13 rat brains

\begin{tabular}{|llll|}
\hline Gene & Fold & Chr & Position (strand) \\
\hline Kdm6c & 134.23 & Y & $1,065,274-1,206,127(-)$ \\
\hline SRY4 & 3.56 & Y & $327,176-327,685(+)$ \\
\hline Olig1 & 1.82 & 11 & $31,428,377-31,430,519(+)$ \\
\hline Corin & -3.07 & 14 & $38,247,379-38,474,931(+)$ \\
\hline Pax7 & -3.60 & 5 & $158,211,001-158,313,510(-)$ \\
\hline Crabp1 & -3.89 & 8 & $59,344,097-59,352,135(+)$ \\
\hline AABR07021574.1 & -5.71 & 13 & $79,272,728-79,273,271(-)$ \\
\hline Xist & -27.36 & $\mathrm{X}$ & $74,324,637-74,342,152(-)$ \\
\hline
\end{tabular}

(C) Identified sexually dimorphic key gene expressed in E14 rat brains

\begin{tabular}{|llll|}
\hline Gene & Fold & Chr & Position (strand) \\
\hline Kdm6c & 199.86 & Y & $1,065,274-1,206,127(-)$ \\
\hline SRY4 & 3.30 & Y & $327,176-327,685(+)$ \\
\hline Fam111a & 2.70 & 1 & $229,003,778-229,019,532(+)$ \\
\hline Zfp871 & 2.31 & 7 & $15,024,693-15,027,933(-)$ \\
\hline
\end{tabular}




\begin{tabular}{|lccc|}
\hline \multicolumn{4}{|c|}{ (A) Identified sexually dimorphic key gene expressed in E12 rat brains } \\
\hline Gpsm3 & 2.17 & 20 & $4,355,246-4,357,107(+)$ \\
\hline Ace & -2.43 & 10 & $94,170,766-94,213,831(+)$ \\
Xist & -46.70 & $X$ & $74,324,637-74,342,152(-)$ \\
\hline
\end{tabular}

\section{Confirmation of RNA seq data by qPCR}

qRT-PCR analysis was performed on Sry4, Ddx3x, Ddx3y, Xist, Kdm6c, Polysaccharide Biosynthesis Domain Containing 1 (Pbdc1), and Eif2s3 to confirm RNA seq data (Figure 3). The qRT-PCR analysis confirmed that there were sex specific differences in gene expression of these genes, in agreement with the data obtained from the RNA sequencing.

\section{Pathway Analysis of Differentially Expressed Genes and Gene Ontology}

Following identification of differentially expressed genes, we performed a pathway enrichment analysis to determine the main affected signaling pathways (Table S2). In E12 brains, three pathways were significantly enriched in males, whereas six were significantly enriched in females. Of these the Rap1 signaling pathway was affected in both males and females at E12 and continued to be enriched in females at E13. The PI3K-AKT signaling pathway showed enrichment in female rat brains at both E12 and E13 as well as in males at E12. At E14, the insulin signaling pathway showed the strongest enrichment in the male brain, while the Ribosome pathway was highly enriched in female brains.

Analysis of GO enrichment data revealed that 467 and 535 biological processes were altered in the E12 male and female brains, respectively (Figure S1). At E13, 474 and 2076 biological processes were altered in male and female brains, respectively. At E14, 495 and 592 biological processes were altered in male and female brains, respectively. At E12, the main GO function was associated with intracellular membrane-bound organelles, including the nucleus. In females at E12 and in both males and females at E13 and E14 the results are more complex with several GO functions being altered as listed in Figure S1, Supplementary Tables 4, 5, 6).

\section{Discussion}

Differential patterning of male and female brains has been suggested to be regulated by steroid hormones released by the gonads. However, studies on zebra finch (Arnold 2003), mouse (Dewing et al. 
2003) and chicken (Lee et al. 2009) suggests an involvement of genetic signals in the development of male and female brain neuronal networks.

In the present study, we have used isolated brain samples to avoid any signal from other head tissues. We selected three embryonic stages to determine sex differences in genetic signaling in the brain, prior to gonadal activation (E12), at the time of gonadal activation (E13), and following gonadal activation (E14). RNA sequencing analysis indicated that the rat brain shows sexually dimorphic gene expression already at the first studied stage, at E12 which is prior to the initiation of gonadal differentiation that occur at around stage E13 (Schulz et al. 2019). The rat gonadal differentiation process initiates with a bipotential gonad, and after E13 it further differentiates either into testis or ovary (Val et al. 2003).

Sry is the master switch in determining mammalian gonadal sex. Expression of Sry in mice testis begins at $10.5 \mathrm{dpc}$ and peaks at $11.5 \mathrm{dpc}$ and by $12.5 \mathrm{dpc}$ the expression is undetectable (Larney et al. 2014). However, Sry has been reported to be expressed in adult mouse and human male brains (Mayer et al. 1998, Vawter et al. 2004, Dewing et al. 2006, Czech et al. 2012). The rat genome contains multiple Sry copies (Prokop et al. 2013). Analysis of Sry expression in rat testis from E12 to E14 identified Sry4A as the top candidate testis-differentiating gene (Schulz et al. 2019). In male rat gonads, Sry4A expression peaks at E13 (Bhandari et al. 2011). However, at this time point the gonad is still underdeveloped (Val et al. 2003). Hence, it can be assumed that steroid secretion at E13 is still not initiated from the testis. Thus, it can be concluded that the differential gene expression profiles observed for rat brain at E12 occur prior to hormone action on the brain.

Of the many rat Sry genes, Sry 4 is the gene that most closely resembles Sry4A (Prokop et al. 2013). The main difference between these two genes is a P83S amino acid replacement in Sry4 and a ten Q insertion after amino acid 154 in Sry $4 A$ (Prokop et al. 2013). While Sry $4 A$ was the top candidate for sex determination in rat testis, Sry 4 was not expressed in testis (Prokop et al. 2020). In the present study, we observed sex differences in brain gene expression of Sry 4 at all three developmental stages, while Sry $4 A$ was not differentially expressed. As Sry 4 was the only Sry gene expressed in the developing brain, this suggests that Sry 4 may be involved in regulating brain sex differentiation.

In the mouse study by Dewing and co-worker, microarray was performed on whole head of $10.5 \mathrm{dpc}$ and 51 differentially regulated genes were identified (Dewing et al. 2003). Of these, the two genes with the highest fold differences were Dby $(D d x 3 y)$ and Eif2s $3 y$, both located on the $Y$ chromosome. While the remaining genes indicated to be differentially expressed were not located to the $Y$ chromosome, one was located to the $\mathrm{X}$ chromosome. In the present study, we identified a larger number of genes as male biased at all three timepoints. The 3 genes showing the highest male bias at E12 were located on the $Y$ chromosome. Two of these genes, $D d x 3 y$ and $K d m 6 c$, are located in the AZFa region of the $Y$ chromosome. This region also contains the Usp9y gene that was not identified in the present study. The $A Z F a, b$ and $c$ regions on the $Y$-chromosome have been identified to be required for normal spermatogenesis (Vog et al. 1996). In the AZFa region it has been suggested that $D d x 3 y$ is the main gene responsible for infertility (Foresta et al. 2000). In male gonads, $D d x 3 y$ is expressed in spermatogonia 
before meiosis and $D d x 3 x$ is expressed in spermatids (Rauschendorf et al. 2014). Deletion of $D d x 3 y$ disrupts germ cell development and leads to infertility in males (Ramathal et al. 2015). The peak expression of $D d x 3 y$ at E12 in rat brain indicate that this gene may also have key functions in male brain development.

The second gene that showed high expression in male rat brains was $K d m 6 c$. The $\mathrm{X}$ chromosome homologue $\mathrm{Kdm} 6 \mathrm{a} / \mathrm{Utx}$ has been shown to be an active demethylase, acting on Lys 27 of histone $\mathrm{H} 3$ (H3K27). Kdm6a/ Utx was also differentially regulated in mouse whole head (Dewing et al. 2003). A recent study indicates that $K d m 6 a$ can sense oxygen availability to regulate chromatin and cell fate (Chakraborty et al. 2019). Apart from being involved in embryonic development and cell differentiation, KDM6A has been shown to regulate tumor growth (Schulz et al. 2019). KDM6A can promote estrogen receptor (ER) positive breast cancer cells and ER can transactivate KDM6A. Hence, KDM6A/UTX forms a feed forward loop with ER (Xie et al. 2017). KDM6C shows $>88 \%$ similarity with KDM6A and has recently been shown to be an active demethylase, demethylating H3K27, but with lower activity (Walport et al. 2014).

\section{Perspective and Significance}

In the present study, we show that the top candidate genes, $D d x 3 y$ and $K d m 6 c$, that are involved in male brain development are expressed in a sexually dimorphic pattern at stage E12, prior to the initiation of gonadal differentiation that occurs at E13 in rat (Clement et al. 2007, Prokop et al. 2020). Here we show that the expression of $K d m 6 c$ remain high at E13 and E14, while $D d x 3 y$ peak at E12. Thus, it is possible that $D d x 3 y$ may play a role in the initiation of brain sex differentiation prior to the initiation of gonadal differentiation. Previous studies have shown that genes encoded by the $Y$ chromosome can result in differences in behavior and brain phenotypes in mice (van Abeelen 1988, van Abeelen et al. 1989). The expression of Sry at all three timepoints suggests that locally expressed Sry may be involved in male brain differentiation. Furthermore, two candidate genes, $D d x 3 y$ and $K d m 6 c$ in the AZFa region of the $Y$ chromosome, were also identified as possible regulators of dimorphic rat brain differentiation prior to gonadal sex differentiation.

\section{Declarations}

\section{Ethics approval and consent to participate}

Not applicable

\section{Consent for publication}

Not applicable 


\section{Availability of data and materials}

Transcriptomics datasets are deposited to the NCBI-Sequence Read Archive (SRA): SRR17237889 (BioProject ID: PRJNA789225), SRR17245042 (BioProject ID: PRJNA789239), SRR17245243 (BioProject ID: PRJNA789412), SRR17245515 (BioProject ID: PRJNA789422), SRR17245590 (BioProject ID: PRJNA789427), SRR17245822 (BioProject ID: PRJNA789436), SRR17245883 (BioProject ID: PRJNA789438), SRR17245899 (BioProject ID: PRJNA789439), SRR17246623 (BioProject ID: PRJNA789448), SRR17246640 (BioProject ID: PRJNA789456), SRR17246808 (BioProject ID: PRJNA789457), SRR17246824 (BioProject ID: PRJNA789461), SRR17247042 (BioProject ID: PRJNA789463), SRR17247071 (BioProject ID: PRJNA789468), SRR17247298 (BioProject ID: PRJNA789470), SRR17247424 (BioProject ID: PRJNA789481), SRR17247536 (BioProject ID: PRJNA789484), and SRR17247560 (BioProject ID: PRJNA789488).

\section{Competing interests}

Authors declare that there is no conflict of interest.

\section{Funding}

This study was financed by the Swedish Research Council and Örebro University.

\section{Authors' contributions}

AP - design, analysis, interpretation, drafting of the manuscript and approval of the submitted version SP - analysis, interpretation, drafting of the manuscript and approval of the submitted version PEO - conception, design, interpretation, funding acquisition, revision of the manuscript and approval of the submitted version

\section{Acknowledgements}

We are grateful to Dr. Carina Modig for proof reading of the manuscript.

\section{References}

1. Arnold, A. P. (2003). "The gender of the voice within: the neural origin of sex differences in the brain." Curr Opin Neurobiol. 13(6): 759-764. 
2. Arnold, A. P. (2009). "The organizational-activational hypothesis as the foundation for a unified theory of sexual differentiation of all mammalian tissues." Horm Behav. 55(5): 570-578.

3. Bhandari, R. K., I. Sadler-Riggleman, T. M. Clement and M. K. Skinner (2011). "Basic helix-loop-helix transcription factor TCF2 1 is a downstream target of the male sex determining gene SRY." PLOS ONE 6(5): e19935.

4. Chakraborty, A. A., T. Laukka, M. Myllykoski, A. E. Ringel, M. A. Booker, M. Y. Tolstorukov, Y. J. Meng, S. R. Meier, R. B. Jennings, A. L. Creech, Z. T. Herbert, S. K. McBrayer, B. A. Olenchock, J. D. Jaffe, M. C. Haigis, R. Beroukhim, S. Signoretti, P. Koivunen and W. G. Kaelin, Jr. (2019). "Histone demethylase KDM6A directly senses oxygen to control chromatin and cell fate." Science 363(6432): 1217-1222.

5. Clement, T. M., M. D. Anway, M. Uzumcu and M. K. Skinner (2007). "Regulation of the gonadal transcriptome during sex determination and testis morphogenesis: comparative candidate genes." Reproduction 134(3): 455-472.

6. Czech, D. P., J. Lee, H. Sim, C. L. Parish, E. Vilain and V. R. Harley (2012). "The human testisdetermining factor SRY localizes in midbrain dopamine neurons and regulates multiple components of catecholamine synthesis and metabolism." J Neurochem. 122(2): 260-271.

7. Dewing, P., C. W. Chiang, K. Sinchak, H. Sim, P. O. Fernagut, S. Kelly, M. F. Chesselet, P. E. Micevych, K. H. Albrecht, V. R. Harley and E. Vilain (2006). "Direct regulation of adult brain function by the malespecific factor SRY." Curr Biol. 16(4): 415-420.

8. Dewing, P., T. Shi, S. Horvath and E. Vilain (2003). "Sexually dimorphic gene expression in mouse brain precedes gonadal differentiation." Brain Res Mol Brain Res. 118(1-2): 82-90.

9. Foresta, C., A. Ferlin and E. Moro (2000). "Deletion and expression analysis of AZFa genes on the human Y chromosome revealed a major role for DBY in male infertility." Hum Mol Genet. 9(8): 11611169.

10. Gahr, M. and R. Metzdorf (1999). "The sexually dimorphic expression of androgen receptors in the song nucleus hyperstriatalis ventrale pars caudale of the zebra finch develops independently of gonadal steroids." J Neurosci. 19(7): 2628-2636.

11. Ingalhalikar, M., A. Smith, D. Parker, T. D. Satterthwaite, M. A. Elliott, K. Ruparel, H. Hakonarson, R. E. Gur, R. C. Gur and R. Verma (2014). "Sex differences in the structural connectome of the human brain." PNAS 111(2): 823-828.

12. Koopman, P. (2005). "Sex determination: a tale of two Sox genes." Trends Genet. 21(7): 367-370.

13. Larney, C., T. L. Bailey and P. Koopman (2014). "Switching on sex: transcriptional regulation of the testis-determining gene Sry." Development 141(11): 2195-2205.

14. Lee, S. I., W. K. Lee, J. H. Shin, B. K. Han, S. Moon, S. Cho, T. Park, H. Kim and J. Y. Han (2009). "Sexually dimorphic gene expression in the chick brain before gonadal differentiation." Poult Sci. 88(5): 1003-1015.

15. Mayer, A., G. Lahr, D. F. Swaab, C. Pilgrim and I. Reisert (1998). "The Y-chromosomal genes SRY and ZFY are transcribed in adult human brain." Neurogenetics 1(4): 281-288. 
16. Phoenix, C. H., R. W. Goy, A. A. Gerall and W. C. Young (1959). "Organizing action of prenatally administered testosterone propionate on the tissues mediating mating behavior in the female guinea pig." Endocrinology 65: 369-382.

17. Prokop, J. W., S. B. Chhetri, J. E. van Veen, X. Chen, A. C. Underwood, K. Uhl, M. R. Dwinell, A. M. Geurts, S. M. Correa and A. P. Arnold (2020). "Transcriptional analysis of the multiple Sry genes and developmental program at the onset of testis differentiation in the rat." Biol Sex Differ. 11(1): 1-8.

18. Prokop, J. W., A. C. Underwood, M. E. Turner, N. Miller, D. Pietrzak, S. Scott, C. Smith and A. Milsted (2013). "Analysis of Sry duplications on the Rattus norvegicus Y-chromosome." BMC Genom. 14(1): 1-16.

19. Ramathal, C., B. Angulo, M. Sukhwani, J. Cui, J. Durruthy-Durruthy, F. Fang, P. Schanes, P. J. Turek, K. E. Orwig and R. Reijo Pera (2015). "DDX3Y gene rescue of a Y chromosome AZFa deletion restores germ cell formation and transcriptional programs." Sci Rep. 5: 15041.

20. Rauschendorf, M. A., J. Zimmer, C. Ohnmacht and P. H. Vogt (2014). "DDX3X, the X homologue of AZFa gene DDX3Y, expresses a complex pattern of transcript variants only in the male germ line." Mol Hum Reprod. 20(12): 1208-1222.

21. Schmittgen, T. D., and Livak, K.J (2008). "Analyzing real-time PCR data by the comparative $C_{T}$ method." Nat Protoc. 3(6): 1101-1108.

22. Schulz, W. A., A. Lang, J. Koch and A. Greife (2019). "The histone demethylase UTX/KDM6A in cancer: Progress and puzzles." Int J Cancer 145(3): 614-620.

23. Sim, H., A. Argentaro and V. R. Harley (2008). "Boys, girls and shuttling of SRY and SOX9." Trends Endocrinol Metab. 19(6): 213-222.

24. Val, P., A.-M. Lefrançois-Martinez, G. Veyssière and A. Martinez (2003). "SF-1 a key player in the development and differentiation of steroidogenic tissues." Nucl Recept. 1(1): 8.

25. van Abeelen, J. H. (1988). "Autosomal and heterosomal influences on behavioral and somatic traits in mice." Behav Genet. 18(1): 99-104.

26. van Abeelen, J. H. F., C. J. J. G. Janssens, W. E. Crusio and W. A. J. G. Lemmens (1989). "YChromosomal effects on discrimination learning and hippocampal asymmetry in mice." Behav Genet. 19(4): 543-549.

27. Vawter, M. P., S. Evans, P. Choudary, H. Tomita, J. Meador-Woodruff, M. Molnar, J. Li, J. F. Lopez, R. Myers, D. Cox, S. J. Watson, H. Akil, E. G. Jones and W. E. Bunney (2004). "Gender-Specific Gene Expression in Post-Mortem Human Brain: Localization to Sex Chromosomes." Neuropsychopharmacology 29(2): 373-384.

28. Vog, P., A. Edelmann, S. Kirsch, O. Henegariu, P. Hirschmann, F. Kiesewetter, F. Köhn, W. Schill, S. Farah and C. Ramos (1996). "Human Y chromosome azoospermia factors (AZF) mapped to different subregions in Yq11." Hum Mol Genet. 5(7): 933-943.

29. Wade, J. and A. P. Arnold (1996). "Functional testicular tissue does not masculinize development of the zebra finch song system." PNAS 93(11): 5264-5268. 
30. Walport, L. J., R. J. Hopkinson, M. Vollmar, S. K. Madden, C. Gileadi, U. Oppermann, C. J. Schofield and C. Johansson (2014). "Human UTY(KDM6C) is a male-specific N-methyl lysyl demethylase." J Biol Chem. 289(26): 18302-18313.

31. Xie, G., X. Liu, Y. Zhang, W. Li, S. Liu, Z. Chen, B. Xu, J. Yang, L. He, Z. Zhang, T. Jin, X. Yi, L. Sun, Y. Shang and J. Liang (2017). "UTX promotes hormonally responsive breast carcinogenesis through feed-forward transcription regulation with estrogen receptor." Oncogene 36: 5497.

32. Xu, H., F. Wang, Y. Liu, Y. Yu, J. Gelernter and H. Zhang (2014). "Sex-biased methylome and transcriptome in human prefrontal cortex." Hum Mol Genet. 23(5): 1260-1270.

\section{Figures}




\section{Figure 1}

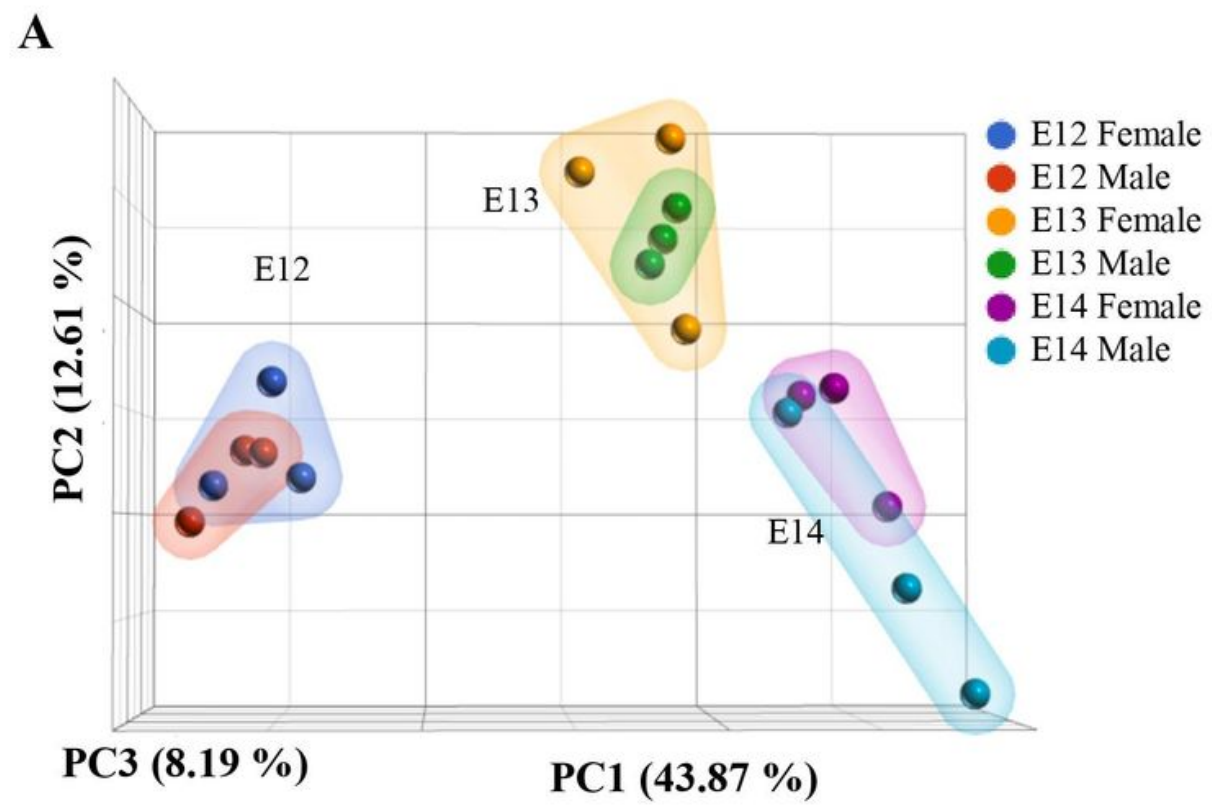

B

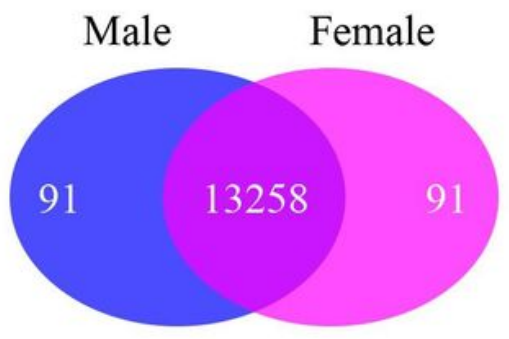

C

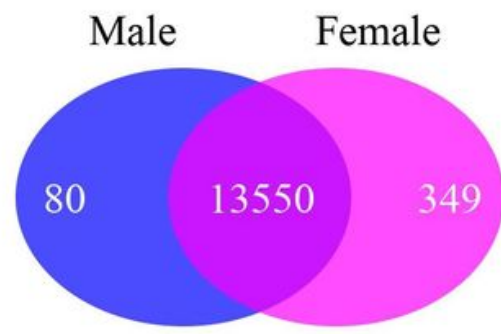

D

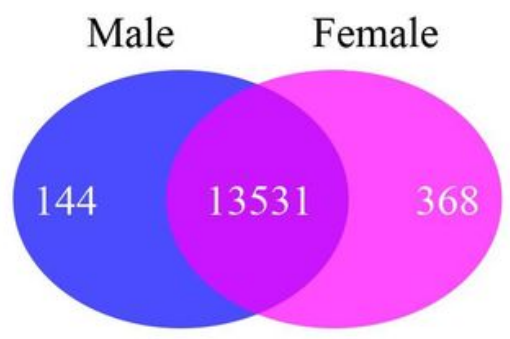

\section{Figure 1}

Differentially gene expression analysis in E12, E13, and E14 rat brains. (A) PCA showed gene expression clustering among the three samples in the male and females' brains of E12, E13, and E14. Within each embryonic stage, dimorphic gene expressions are prominent and form sub-clusters in the male and female brains. Number of genes expressed in males and females of E12 (B), E13 (C), and E14 (D) brains are shown in the blue and magenta ellipses, respectively. Whereas the number of genes expressed in both males and females of E12 (B), E13 (C), and E14 (D) brains are shown in overlapping regions. 


\section{Figure 2}

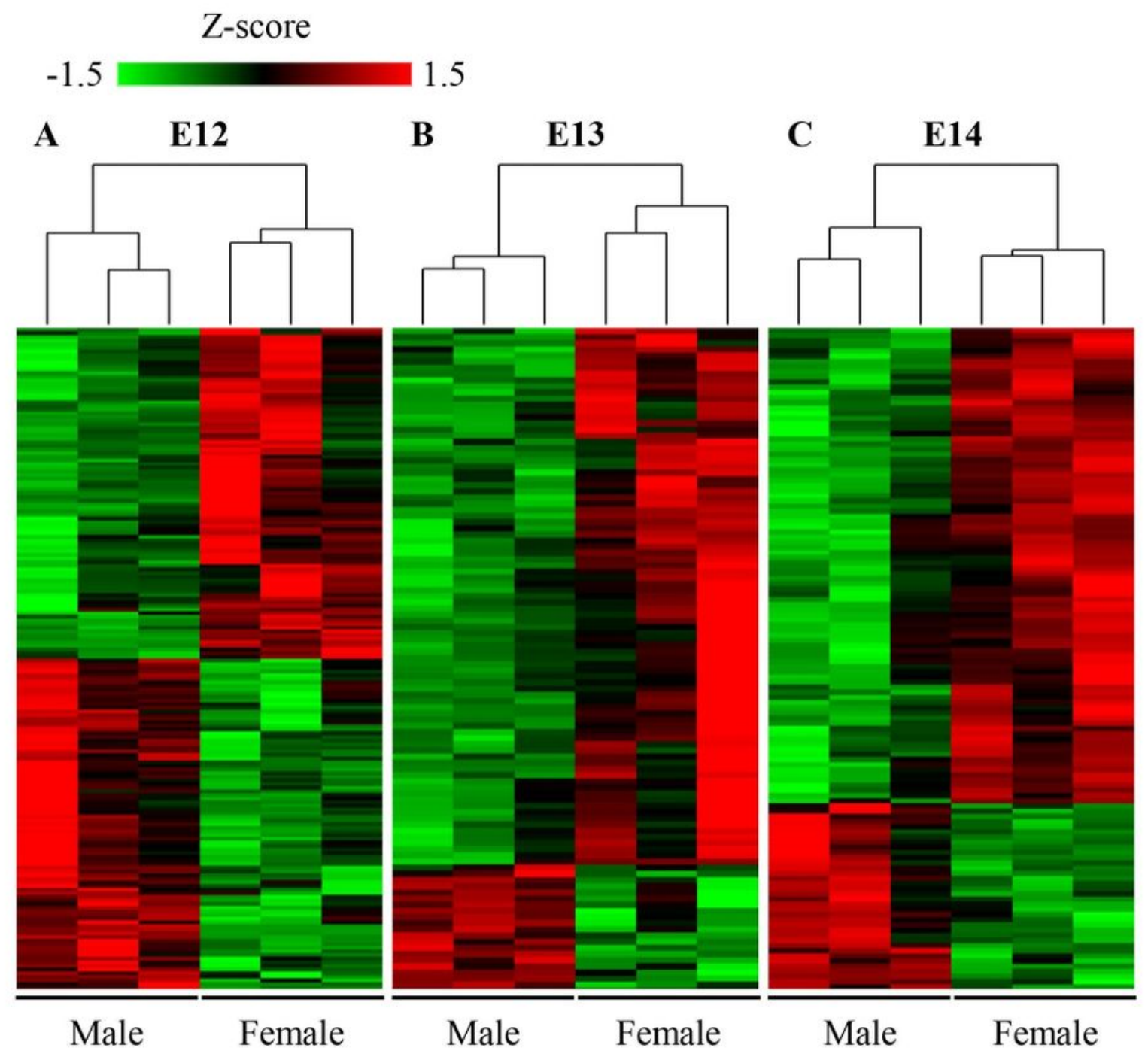

\section{Figure 2}

Heat map of the differentially upregulated genes. The expression patterns at the three studied developmental stages showed that there were of 91 upregulated genes in E12 males and females, respectively (A), 80 upregulated genes in E13 males and 349 upregulated genes in E13 females (B), and 144 upregulated genes in E14 males and 368 upregulated genes in E14 females (C). Red denotes upregulation and green downregulation. 
Figure 3
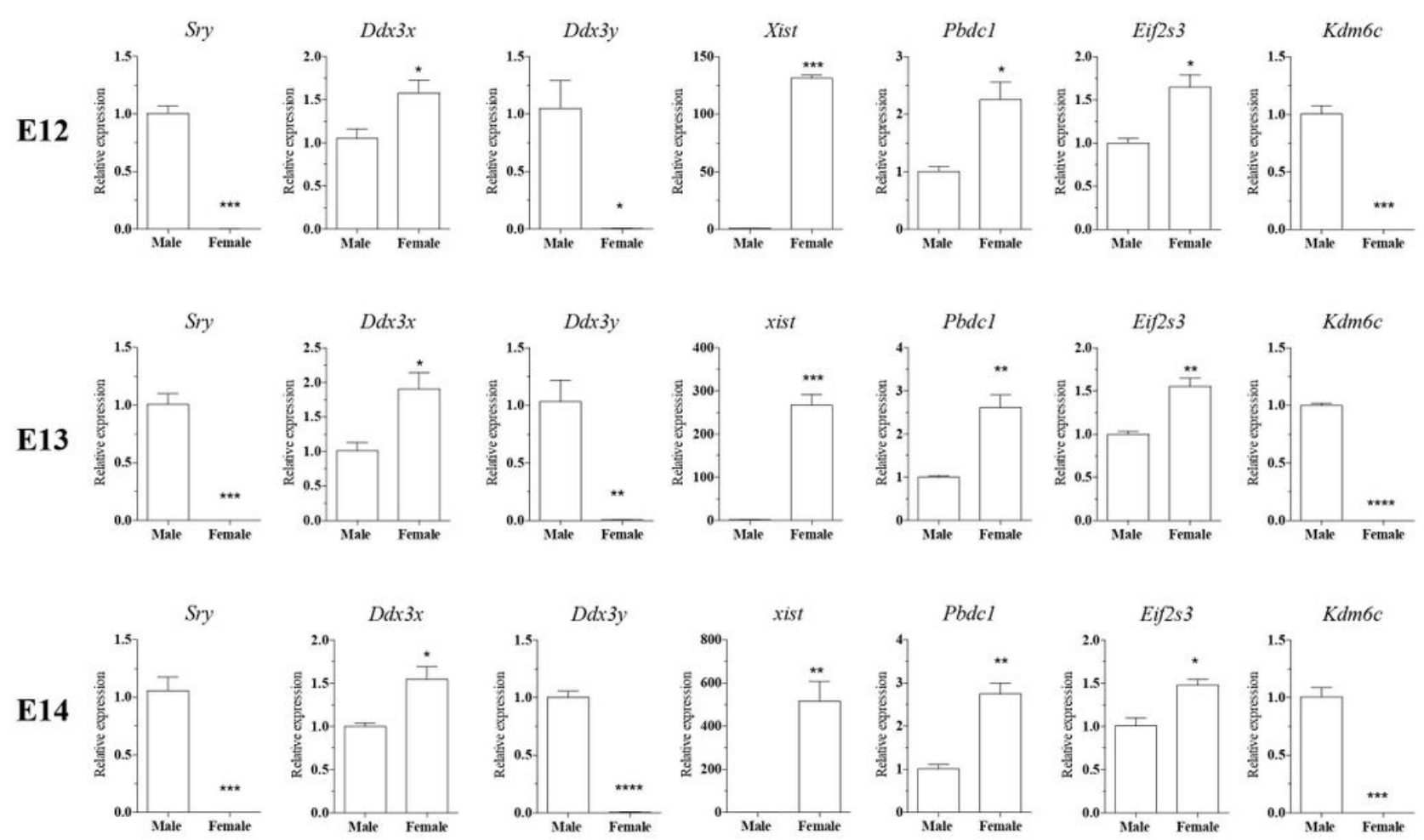

Figure 3

Validation of gene expression patterns using qRT-PCR. In order to confirm the differential expression patterns a qRT-PCR analysis was performed on a selection of genes with different expression patterns. This analysis confirmed the the expression patterns of the analysed genes (Sry, Ddx3y, Xist, Kdm6c, $P b d c 1$, and Eif2s3) as observed in the RNA sequencing data.

\section{Supplementary Files}

This is a list of supplementary files associated with this preprint. Click to download. 
- ManuscriptSupplementarydata20211209.docx

- SupplementaryTable1.xlsx

- SupplementaryTable2.xIsx

- SupplementaryTable3.xlsx

- SupplementaryTable4.xIsx

- SupplementaryTable5.xlsx

- SupplementaryTable6.xlsx 